\title{
Récits de femmes - Analyse multimodale du récit conversationnel en français : une étude de cas
}

\author{
Gaëlle Ferré \\ LLING - Université de Nantes \\ Chemin de la Censive du Tertre, BP 81227 \\ 44312 Nantes cedex 3 \\ Gaelle.Ferre@univ-nantes.fr
}

\section{Introduction}

Ainsi que l'observe J. Coates (1997, 2001), les récits personnels jouent un rôle essentiel dans la conversation spontanée. La plupart du temps, il ne s'agit pas tant pour celui qui raconte d'informer son interlocuteur de faits réels tirés de son expérience personnelle ${ }^{1}$, que de maintenir le lien social en donnant à la conversation un caractère ludique :

\footnotetext{
"The main goal of talk-at-play is the construction and maintenance of good social relations, not the exchange of information [...]. The second goal of talk-at-play is that participants should enjoy themselves. The fun of talk arises as much from how things are said as from what is said." (Coates, $1997: 85$ )
}

En ce qui concerne les récits d'expériences négatives et/ou extrême, ils permettent au narrateur de se soulager d'une charge émotionnelle trop lourde à porter. Ceci explique largement pourquoi les récits personnels se retrouvent en si grand nombre dans la conversation spontanée, mais aussi pourquoi les études portant sur le récit ont connu un essor considérable depuis un demi-siècle, favorisées par le développement d'enregistrements audio d'interactions spontanées. Au croisement de la sociologie et de l'analyse de discours, Labov \& Waletzky (1967), et plus tard Labov $(1997,2007)$ ont réalisé une étude pionnière sur la structure verbale interne de récits en anglais, élicités et enregistrés par les chercheurs. Ce travail a constitué le cadre pour de nombreux chercheurs travaillant sur le récit dans un grand nombre de domaines scientifiques comme la typologie, l'analyse de discours, l'acquisition des langues en linguistique (Chafe, 1997 ; Ochs, 1997 ; Ochs \& Capps, 1997 parmi d'autres), mais aussi l'ethnographie, la psychologie, la sociologie par exemple (l'ouvrage édité par Bamberg, 2007, contient d'ailleurs une collection d'articles inscrits dans une pluralité de champs scientifiques). C'est également dans ce cadre que s'inscrit le présent article sur le récit en français, qui propose une contribution à l'analyse des récits dans le champ de l'analyse du discours. En fait, si la plupart des études sur le récit reposent sur des enregistrements audio de récits en conversation spontanée, la majeure partie des travaux réalisés jusqu'à présent se sont essentiellement concentrés sur la structure verbale des séquences narratives. Même si leur apport est considérable, il me semble que l'on peut approfondir les connaissances sur la structure narrative en tenant compte des paramètres liés à l'oralité.

Ainsi, si l'on considère l'interaction elle-même, et non pas le point de vue de l'analyste sur cette interaction, ainsi que l'a souligné récemment Local (2007), l'information est traitée en direct par les participants qui l'encodent et la décodent au moyen de paramètres acoustiques, paramètres qui devraient par conséquent être pris en compte par l'analyste. Dans son article qui portait sur l'organisation des tours de parole en anglais, Local n'envisage que les paramètres acoustiques puisque son travail reposait essentiellement sur l'enregistrement de conversations téléphoniques. Tout en approuvant le fait que les recherches sur la conversation devraient porter une attention plus grande aux traits acoustiques, j'étendrais néanmoins la proposition de Local puisque les participants ne sont pas uniquement des locuteurs, mais sont aussi des acteurs de l'interaction. Ces acteurs utilisent également des indices visuels pour organiser l'interaction, indices qu'il serait dommage de laisser de côté dans les analyses. C'était d'ailleurs le point de vue adopté par C. \& M.H. Goodwin, dès les années 80 (Goodwin, C., 1984, 1986 ; Harness Goodwin, 1982, 1997 ; Goodwin \& Harness Goodwin, 1992), dans une série d'articles sur le 
récit. Dans ces articles, ils s'inspirent de la typologie labovienne du récit et montrent comment le récit est co-construit dans des conversations à plusieurs participants. La démarche était cependant différente de celle qui est proposée ici. C. et M.H. Goodwin ont montré comment lors de la production d'un récit, les participants à la conversation s'orientent les uns envers les autres à travers leur discours, mais aussi à travers leur gestualité. Bien que la prosodie soit évoquée (comme par exemple le débit de parole, la présence de pauses), ils ne proposent pas une analyse prosodique systématique (en termes de contours prosodiques, par exemple) et outillée des récits étudiés. En ce qui concerne la gestualité, leurs études se concentrent sur le regard et l'orientation de la tête et du corps des participants (paramètres essentiels pour la compréhension du fonctionnement de l'interaction), mais ils ne pouvaient alors utiliser des typologies comme celle mise en place par McNeill (1992) pour les gestes manuels, par exemple, et qui offre une première analyse de la relation existant entre le geste et le discours.

L'étude que je présente ici est basée sur des enregistrements vidéo de conversations entre des Françaises, décrits dans la section 2. L'hypothèse présentée dans la section 3 est que les paramètres auditifs et visuels $^{2}$ utilisés par les participants pour structurer leurs récits concordent assez largement avec la structure sémantique et syntaxique canonique du récit décrite par Labov. Cependant, lorsque le contenu sémantique du récit ne permet pas de décoder sa structure, les indices prosodiques et visuels ajoutent des informations qui peuvent se révéler utiles dans l'analyse.

Les recherches sur le récit inscrites dans une perspective multimodale ${ }^{3}$ sont cependant relativement récentes et encore assez peu développées (Bouvet \& Morel, 2002 ; McNeill, 1992, 2001 ; McNeill et al., 2001 a et $b$, ainsi que d'autres travaux réalisés au Laboratoire de Linguistique et de Psychologie de Chicago - CENTER FOR GESTURE AND SPEECH RESEARCH), ce qui ne nous permet pas à l'heure actuelle d'effectuer des comparaisons entre études basées sur de grands corpus de conversations spontanées (les travaux menés par McNeill et son équipe sont d'ailleurs basés sur des récits élicités après vivionnement d'un dessin animé par les participants). Les travaux cités ne s'inscrivent pas non plus dans la perspective labovienne qui est adoptée ici. C'est pourquoi j'ai préféré proposer ici une étude de cas plutôt qu'une étude de type quantitatif. Mon souci est de ne pas effectuer de généralisations prématurées sur un trop petit nombre de cas. Néanmoins, si un seul exemple est présenté dans sa globalité dans cet article, les observations initiales ont été réalisées sur un plus grand nombre de récits et les paramètres retenus dans ce travail reposent sur des calculs et des observations des paramètres linguistiques à l'oeuvre dans l'interaction.

\section{Les données}

Cette étude n'est pas une étude de genre (gender study) bien qu'elle repose sur des enregistrements de conversations entre femmes. Coates (2001) a montré que les récits produits par les femmes étaient très différents de ceux produits par les hommes dans leur contenu sémantique : selon elle, les récits produits par les hommes contiennent de plus amples détails physiques dans leurs descriptions des objets et des événements. Leurs récits tournent également plus autour de la compétition et de la réussite. Leur langage serait plus grossier. Enfin, les hommes afficheraient moins que les femmes leurs émotions. Coates montre également que les hommes produisent des récits différents lorsqu'ils se trouvent en compagnie de femmes. Leurs récits sont alors plus collaboratifs. Cette collaboration moindre dans les conversations entre hommes est liée à la présence de taboos sociaux :

[The] capacity of sequential story-telling to testify to the closeness of participants means that it can be a powerful way of "doing" friendship. I argue that this aspect of sequential story-telling has particular value for male speakers who need to be careful how they display connectedness because of the taboos associated with homosexuality.

De plus, la variation intra- et inter-locuteur est déjà importante lorsque l'on analyse les paramètres prosodiques et l'est plus encore si l'on y ajoute les paramètres gestuels. Le présent article fait suite à un travail précédent du même auteur (Ferré, 2005) dans lequel une analyse multimodale avait été proposée pour des récits produits par des locutrices anglaises dans lequel des premières observations avaient été effectuées. Dans l'optique d'une continuité dans l'analyse du récit, il a donc semblé souhaitable de comparer des récits produits par des participants du même sexe, mais aussi du même âge environ. 


\subsection{Le corpus CID}

Le corpus qui a été utilisé pour ce travail est le Corpus of Interactional Data, enregistré au Laboratoire Parole et Langage d'Aix en Provence et qui a été décrit dans le détail dans Bertrand et al. (2006). Les enregistrements vidéo ont été réalisés par R. Bertrand et B. Priego-Valverde, et consistent en plusieurs heures de dialogues entre pairs (une heure de conversation par enregistrement). Les participants ont été filmés dans une chambre sourde. Ils se connaissaient bien et étaient familiers des lieux et des techniques d'enregistrement, ceci afin de garantir un plus grand naturel dans les interactions. Ils étaient assis l'un à côté de l'autre, étaient filmés par une seule caméra et enregistrés par deux micros sur deux pistes son séparées pour une meilleure qualité du signal audio (nécessaire pour réaliser des analyses acoustiques). Le fait que les participants aient été assis côte-à-côte est important car cela permet de mieux cerner leur orientation: en effet, dans une conversation en face-à-face, il est difficile de savoir si le participant oriente son corps vers l'autre à des fins interactionnelles ou s'il adopte simplement la position de repos (par exemple, la tête dans l'orientation du reste du corps), $c f$. Sacks \& Schegloff (2002). Lorsque les participants sont assis côte-à-côte, la tête orientée vers l'autre n'est pas en position de repos, ce qui témoigne d'un certain engagement conversationnel.

Le corpus a été transcrit dans sa globalité et de nombreuses annotations ont été réalisées dans plusieurs champs linguistiques, notamment la phonétique/phonologie, la morphologie, la syntaxe, la prosodie. La recherche comprend également l'annotation des mouvements et des gestes réalisés par les participants. Cette annotation doit se faire de manière entièrement manuelle, aussi est-elle particulièrement longue et encore inachevée. Malgré cela, plusieurs études ont déjà pu être réalisées sur le corpus, qui ne portaient cependant pas sur les récits (Bertrand et al., 2007 ; Ferré et al., 2007 ; Portes \& Bertrand, 2006 ; Portes, Bertrand \& Espesser, 2007, pour n'en citer que quelques unes). C'est afin de permettre une exploitation la plus vaste possible que l'enregistrement a été réalisé selon un protocole quasi expérimental. En effet, un enregistrement in situ, réalisé avec une seule caméra et un seul microphone ne permet pas d'effectuer une analyse acoustique fiable (notamment à cause de la présence de bruits de fond et de chevauchements de parole des participants dans de telles conditions). De même, pour une analyse systématique de la gestualité (et notamment des micro-mouvements de la face), l'enregistrement doit garantir un bon éclairage et proscrire une trop grande mobilité des participants qui sinon, risquent de se retrouver de dos à un moment crucial de l'analyse.

Dans ce corpus, j'ai choisi de travailler sur deux conversations d'une heure chacune entre 4 femmes. Malgré la consigne qui leur avait été donnée de raconter des événements insolites dans leur vie, les conversations sont assez libres. Le corpus peut donc être qualifié de spontané par opposition à de la lecture ou à un discours préparé. La tâche donnée aux locuteurs était particulièrement adaptée pour produire des récits conversationnels ${ }^{4}$ qui sont nombreux dans les deux conversations et varient dans leur longueur et dans leur complexité. J'ai donc sélectionné un certain nombre de ces récits afin d'obtenir un échantillon de récits comparables en longueur et en complexité, et qui se démarquent particulièrement des séquences non narratives de la conversation.

\subsection{Identification des récits dans le corpus et étiquetage des phases du récit selon la typologie de Labov}

La première étape du travail a consisté à isoler les récits des séquences non narratives de la conversation à partir de la transcription orthographique. J'ai identifié les séquences narratives en utilisant les critères suggérés par Küntay \& Ervin-Tripp (1997 : 2), c'est-à-dire les décrochements temporels, les marqueurs linguistiques tels que les conjonctions temporelles, mais aussi les préfaces (Sacks, 1995). Lorsque les récits s'enchaînaient sur plusieurs tours de parole produits par les deux participants, j'ai considéré chaque récit comme une unité distincte et indépendante.

L'étiquetage des différentes phases des récits selon la typologie de Labov (op. cit.) a été réalisé par deux auditeurs profanes après une brève présentation de la structure canonique du récit selon Labov. Les auditeurs profanes enseignent à l'université mais ne sont pas linguistes et ne travaillent pas sur le récit 
dans d'autres disciplines. Seuls les récits pour lesquels les auditeurs profanes s'acordaient sur les différentes phases ont été retenus pour l'analyse. La typologie labovienne utilisée était la suivante :

- Abstract : une phase apparaissant au tout début du récit dans laquelle le narrateur mentionne de manière anticipée la chute du récit,

- Orientation: phase dans laquelle le narrateur présente l'acteur principal, le lieu, le temps et la situation générale du récit, ainsi que des éléments qui vont constituer les connaissances partagées des deux interlocuteurs et sur lesquelles reposent le récit,

- Complication : une série d'événements qui mènent à un 'climax' ou une 'apogée', sous-spécifié par Labov (1997: 406) comme 'the most reportable event in the narrative', c'est-à-dire comme l'événement le plus notable du récit,

- Evaluation : phase dans laquelle le narrateur et/ou l'auditeur font un commentaire de type évaluatif qui peut porter soit sur l'événement le plus important du récit, soit sur l'intégralité du récit lui-même,

- Coda : phase dans laquelle le narrateur revient au temps de l'interaction, avec un commentaire de type 'et voilà'.

Je puis d'ores et déjà préciser qu'aucun des récits observés sur le corpus ne présentaient d'abstract. Rares sont également les récits qui contiennent une coda. Ceci s'explique sans doute par le fait que les récits n’ont pas été directement élicités par un interviewer, mais étaient au contraire insérés dans la conversation de sorte que les participants n'avaient pas l'impression que leur tâche consistait à produire un récit et que celle-ci s'achevait une fois le récit terminé. Leur tâche réelle était de parler avec un ami pendant une heure devant une caméra. Les récits obtenus dans le corpus sont donc tout à fait comparables à des anecdotes racontées en situation naturelle (dans une conversation qui n'aurait pas été provoquée par les chercheurs). En revanche, ils contiennent parfois une «préface », ainsi que la définit Sacks (1995) :

"giving information about what it will take for the story to be over" (1995: 228, Vol. 2)

Ainsi, si un événement terrible est annoncé en préface, le récit s'achève lorsque cet événement terrible a été formulé.

Lerner (1992 : 261) précise que le récit conversationnel est construit selon une trajectoire temporelleséquentielle, ce qui correspond bien aux phases décrites par Labov. En revanche, dans le grand nombre de récits observés, j'ai remarqué que les narrateurs font régulièrement des parenthèses dans la structure narrative. Ils utilisent ces parenthèses pour s'assurer qu'un point du récit fait partie des connaissances partagées avec l'interlocuteur. Les parenthèses se présentent donc souvent sous la forme de questions adressées à l'interlocuteur, dans lesquelles le narrateur s'assure que telle personne à laquelle il est fait allusion ou que tel terme technique employé est connu de l'interlocuteur, ou bien encore que les deux participants à l'interaction sont en accord sur tel ou tel point. Après la parenthèse, le narrateur a deux options : soit il répète ou résume un énoncé déjà produit dans le cas où la parenthèse venait interrompre le déroulement (temporel-séquentiel) narratif, soit il reprend le récit tel qu'il avait été laissé, comme si la parenthèse n'avait pas été faite.

Les auditeurs profanes devaient retrouver les différentes phases des récits dans trois conditions : ils devaient dans un premier temps annoter le script des récits qui étaient découpés en propositions syntaxiques pour une meilleure lisibilité. Ils avaient ensuite la possibilité de modifier leur première annotation après avoir écouté les fichiers son correspondant à chaque récit et enfin après avoir visionné les clips vidéo. Le but de ce test de perception à petite échelle était double : tout d'abord, j'ai fait appel à des auditeurs profanes pour l'annotation des phases du récit pour éviter une certaine circularité dans le raisonnement (ce qui aurait été le résultat obtenu si j'avais moi-même procédé à cette annotation et que dans un second temps, je m'étais attachée à décrire les indices auditifs et visuels observés dans chaque phase). La deuxième raison était de voir si l'interprétation des auditeurs profanes serait modifiée dans les trois conditions (script - écoute - visionnement). Le postulat ici était que si aucune différence n'était observée dans l'étiquetage des phases du récit dans les trois conditions, alors les paramètres auditifs et 
visuels ne joueraient aucun rôle dans l'interprétation des récits. Si en revanche une différence était observée dans l'attribution des phases du récit dans les trois conditions, alors il se pourrait bien que les indices auditifs et visuels, qui viennent en supplément par rapport à la simple lecture d'un script, ajoutent de l'information à cette lecture.

C'est exactement ce qui s'est produit dans ce test pour une phase particulière du récit. En effet, les auditeurs profanes ont étiqueté les phases d'orientation, de complication et d'évaluation dans les récits transcrits et ni l'écoute, ni le visionnement ne les ont incités à modifier leur première impression. Au contraire, pour l'apogée, c'est-à-dire l'événement le plus important du récit, le fait d'écouter et de visionner les enregistrements a amené les auditeurs profanes à modifier leur étiquetage des scripts. Cela signifie que les narrateurs utilisent des indices auditifs et/ou visuels pour exprimer ce qu'ils estiment être important dans leur récit. Et ce qui est important pour eux n'est pas directement accessible dans le contenu sémantique. Même si l'on ne peut savoir exactement quelles ressources - textuelle, visuelle ou sonore - sont mobilisées par les testeurs dans leur étiquetage, le fait qu'ils changent leur étiquetage après avoir visionné le film signifie qu'ils sont sensibles à des indices sonores et/ou visuels, sans pour autant en être nécessairement conscients, et puisqu'un récit conversationnel est fait pour être entendu, la perception des auditeurs devrait être intégrée dans l'analyse. C'est ce qui a été fait dans ce travail puisque j'ai retenu les phases étiquetées dans les conditions audio et vidéo.

\subsection{Annotation prosodique des récits}

Dans un second temps, j'ai extrait les récits du reste des conversations dans Praat et utilisé une transcription en mots alignée avec le son (en utilisant la piste son du narrateur puisque le feedback vocal et verbal de l'interlocuteur n'était pas nécessaire à ce niveau de l'analyse - j'ai utilisé le feedback de l'interlocuteur plus tard dans le traitement vidéo du corpus). Ainsi que le note Lerner (1992: 249) :

\footnotetext{
"For a story to begin and be successfully told recipients must in a sense recognize that a story is about to be told and they must provide the teller with an 'action space' to deliver the story by limiting their participation to actions such as continuers (...) and other forms of story recipiency that sustain the delivery of the story and do not derail it."
}

Bien entendu, la participation de l'interlocuteur ne se limite pas à la simple production de backchannels, mais ceux-ci sont néanmoins utiles pour la détection des phases du récit (voir la section 3.3.)

J'ai également transcrit les récits en propositions syntaxiques et ai utilisé EasyAlign pour le français (développé par J.-P. Goldman, voir les Outils en fin d'article), pour transcrire chaque mot en syllabes. Cette transcription n'avait pas été faite au préalable et était nécesaire pour calculer le débit de parole.

Une fois faites ces transcriptions, j'ai entré dans une piste de Praat les analyses des phases du récit réalisées par les auditeurs profanes.

J'ai ensuite traité chaque récit avec le Prosogram développé par P. Mertens (voir Outils en fin d'article). Cet analyseur m'a semblé particulièrement adapté à cette étude dans la mesure où il propose une stylisation des courbes de F0 qui prend en compte plusieurs paramètres acoustiques comme l'intonation, l'intensité, la durée des segments, et correspond mieux à la perception de l'intonation que la courbe de F0 brute obtenue dans Praat. Dans la mesure où cette étude repose sur la perception des récits, il semblait justifié d'utiliser une stylisation basée sur la perception intégrée des paramètres acoustiques.

A partir des courbes obtenues grâce au Prosogram, j'ai ensuite annoté dans Praat les contours intonatifs globaux. Les contours utilisés étaient Flat (contour plat), High et Low Rise (montée majeure/mineure), High et Low Fall (descente majeure/mineure) ainsi que des contours complexes comme Fall-Rise (descendant-montant) ou Rise-Fall (montant-descendant). J'ai également emprunté le symbole \% au standard d'annotation de l'intonation ToBI, pour indiquer que les montées ou les descentes intonatives se produisaient à la frontière d'un groupe intonatif, c'est-à-dire sur la syllabe précédant immédiatement une frontière de groupe intonatif (par opposition à un contour montant ou descendant graduel sur l'ensemble du groupe intonatif). Enfin, pour chaque groupe intonatif, j'ai noté la hauteur intonative en relation avec 
la fourchette intonative des locutrices sur l'intégralité du récit selon trois niveaux : Top (haut), Mid (moyen) et Bottom (bas).

\subsection{Annotation des gestes}

Après avoir réalisé l'annotation prosodique sur le fichier son, j'ai ensuite effectué l'annotation des gestes et des mouvements effectués par les narrateurs pour chaque récit. J'ai utilisé pour cela le logiciel d'annotation de la vidéo Elan (développé par H. Sloetjes, voir Outils en fin d'article). Après avoir ouvert chaque clip vidéo dans Elan, j'ai importé les pistes d'annotation réalisées sous Praat (Elan étant compatible avec Praat, mais en revanche tout à fait inadapté à la transcription en mots ou en syllabes), à savoir les transcriptions, les contours prosodiques et phases du récit alignés avec la piste son. Dans la mesure où la piste son correspond exactement au fichier vidéo, toutes les annotations réalisées sous Praat sont alignées avec les images et la piste son de la vidéo. L'importation des pistes d'annotation réalisées sous Praat est le fondement même d'une analyse multimodale puisqu'il est alors possible de mettre en relation des annotations effectuées dans différents domaines linguistiques, dans le cas présent la sémantique, la prosodie et les gestes, et de savoir à quel point précis du discours un geste commence et se termine.

Pour l'annotation des gestes, j'ai créé quatre pistes dans Elan : dans les trois premières pistes ont été annotés les mouvements et les gestes réalisées par la narratrice du récit et la dernière piste permettait d'annoter le feedback de l'interlocutrice, qui pouvait être vocal (p.ex. un rire), verbal (backchannels tels que 'ouais', 'mhmh', etc.) ou gestuel (sourire ou hochement de tête). Dans la première piste gestuelle de la narratrice, j'ai annoté les gestes des mains en utilisant la typologie des gestes manuels de McNeill (1992). Seuls les adaptateurs (gestes de contact) ont été ajoutés à cette typologie qui contient les types de geste suivants :

- Iconics : gestes figurant une action, la taille, la forme ou l'emplacement d'un objet,

- Metaphorics : gestes figurant une idée abstraite, exprimant souvent des liens grammaticaux entre deux propositions,

- Emblems : gestes conventionnels,

- Deictics : gestes référentiels,

- Beats : gestes de scansion rythmique pouvant être produits avec la main ou la tête.

Dans la seconde piste gestuelle, j'ai annoté les mouvements de tête de la narratrice (hochements de tête, mouvements de négation, tête inclinée, etc.) ainsi que la direction du regard de la narratrice (vers l'interlocutrice, vers le bas, etc.). Enfin, dans la troisième piste gestuelle, j'ai annoté les sourires et les rires de la narratrice. Pour chaque geste/mouvement produit par les deux participantes, l'annotation commence à la première image où la tête/la main/la bouche quitte sa position de repos et dure jusqu'à la première image où elles retrouvent la position de repos.

Figure 1. Vue de la fenêtre d'annotation de Elan. 


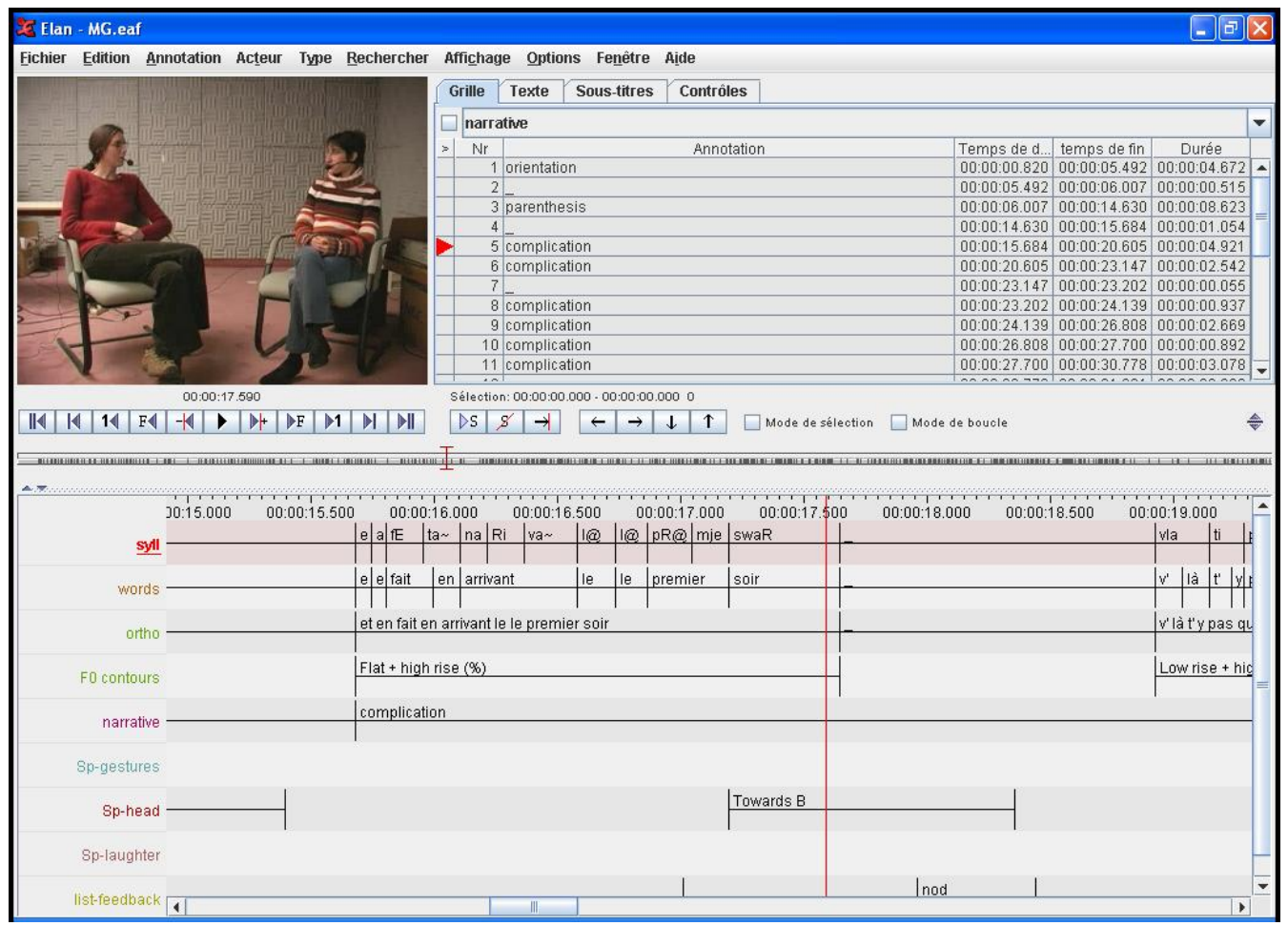

Un aperçu de la fenêtre d'annotation d'Elan est donné dans la Figure 1 ci-dessus. L'annotation des gestes dans Elan a une précision de l'ordre du pixel dans la vidéo et peut donc être mise en relation très précisément avec la piste son et les transcriptions réalisées sous Praat.

En haut à gauche de la Figure 1 apparaît l'image vidéo au temps indiqué par le curseur (la ligne rouge dans la grille d'annotation). Immédiatement sous la vidéo se trouvent les commandes de lecture. En haut à droite de la Figure 1 apparaissent les phases du récit pour l'intégralité du clip, ce qui permet un déplacement rapide d'une phase à une autre car la vidéo s'affiche automatiquement au temps de début de la phase du récit sélectionnée. Sous cette grille se trouvent les commandes d'annotation. Enfin, dans la partie inférieure de la fenêtre apparaissent les annotations. Ici, on voit la transcription de la proposition 'et en arrivant le premier soir', également transcrite en mots et en syllabes (trois premières pistes), qui commence avec un contour intonatif plat et se termine avec une montée de continuation majeure sur 'soir'. Juste avant la production de 'soir', la narratrice (à gauche sur la vidéo) tourne la tête vers l'interlocutrice qui produit un hochement de tête pendant la pause silencieuse qui suit la proposition.

\section{Hypothèse de travail et analyse qualitative d'un exemple}

Ce que nous avons pu observer à partir de l'annotation des données décrite dans la section précédente est que les paramètres auditifs et gestuels sont utilisés par les locutrices pour structurer leurs récits sans doute autant que les indices discursifs tels que les changements de temps, ou l'utilisation de marqueurs discursifs particuliers comme les adverbes temporels ou les connecteurs logiques. On peut également observer que les interlocutrices utilisent ces paramètres pour suivre la progression du récit en cours. On peut même aller plus loin et affirmer qu'à certains points du récit, les indices auditifs et visuels sont prépondérants, particulièrement quand le récit atteint son apogée, qui constitue l'élément central. Par conséquent, l'hypothèse de travail que je propose est que l'apogée du récit est exprimée avec un schéma prosodique et gestuel qui la met en relief par rapport aux autres phases de la narration. Plutôt que de fournir des tableaux de chiffres et des moyennes réalisées sur une large quantité de données, ce qui n'aurait pas vraiment de sens dans une étude d'analyse de discours, je préfère présenter ici une étude de 
cas et montrer l'évolution de l'utilisation de cet ensemble de paramètres dans le déroulement d'un récit dans sa globalité, ce qui permet entre autres de tenir compte du contexte et de l'unité de la narration.

Je donne donc en 3.1 un exemple de récit humoristique qui peut être considéré comme représentatif des récits produits par cette participante dans cette interaction. Connaissant la participante, il m'est même possible de dire que ce récit n'est pas atypique par rapport aux anecdotes qu'elle produit dans des interactions plus 'naturelles' (c'est-à-dire en dehors du laboratoire). Elle le raconte dans une suite de récits où les deux participantes parlent de faits inhabituels dans des appartements qu'elles ont été amenées à louer. Dans l'un de ces appartements, la narratrice raconte avec humour que les propriétaires avaient ôté les lustres, les ampoules, mais aussi les douilles dans chaque pièce, privant ainsi les nouveaux occupants de lumière le soir de leur arrivée. Voici l'analyse structurelle qu'en ont faite les auditeurs profanes après avoir visionné la vidéo :

\subsection{Exemple de récit - M.-L. : les douilles}

\section{[ORIENTATION]}

toujours dans cet appart à Meyreuil là

qu'était super humide

et euh y avait toutes les prises de téléphone sur la même façade

\section{[ABORTED COMPLICATION]}

y avait et ils avaient euh c'é- hum

\section{[ORIENTATION]}

on a- on avait pas fait attention en visitant

d'autant plus que euh on avait visité en plein jour

donc y avait pas la lumière allumée

\section{[COMPLICATION]}

et en fait en arrivant le le premier soir

voilà t'y pas qu'on essaye d'allumer la lumière

puis on bon on arrive comme tout le monde

et on appuie sur l'interrupteur

et $\mathrm{y}$ se passe rien

on se dit merde alors on on on on se débrouille

on regarde un petit peu

et en fait on se rend compte que

ils avaient donc ils avaient enlevé les lustres

et ils avaient aussi enlevé les ampoules

ils avaient aussi enlevé les douilles

[APEX]

$\mathrm{y}$ avait plus rien

y avait plus que les fils qui pendaient

$\mathrm{y}$ avait même plus de quoi poser une ampoule quoi

fallait même qu'on aille jusqu'à racheter les les

\section{[PARENTHESIS]}

c'est ça

c'est les douilles que ça s'appelle les machins pour accrocher les ampoules

\section{[EVALUATION]}

enfin ils avaient enlevé jusqu'à ça quoi

on s'est dit putain mais c'est pas possible quoi

ah ouais non faisaient très très fort

ils étaient super 


\subsection{Paramètres prosodiques dans le récit}

Dans une étude précédente (Ferré, 2005), j’ai montré que trois paramètres prosodiques jouent un rôle récurrent dans la structuration des séquences narratives, soit l'intonation (F0), l'intensité et le débit de parole. Dans l'exemple de récit donné en 3.1, en ce qui concerne l'intonation et l'intensité, la narratrice entame la première phase d'orientation avec une intensité relativement basse et des contours intonatifs situés dans sa plage basse, contours qui ne comportent que de légères montées de continuation en fin de groupe intonatif (sur les montées de continuation, voir Portes \& Bertrand, 2005 ; Portes et al., 2007). Ceci apparaît dans le tracé du Prosogram suivant :

Figure 2. Prosogram de la phase d'orientation du récit.
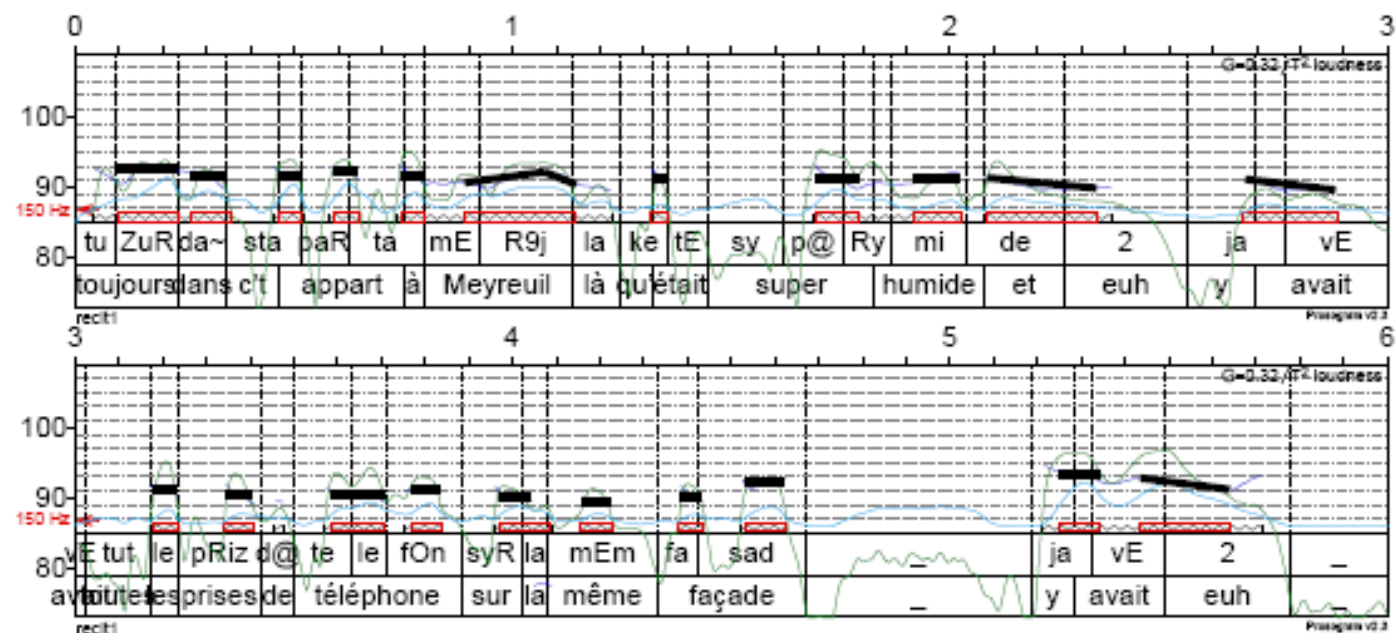

Au cours du développement narratif, l'intensité et la F0 vont progressivement monter vers la plage moyenne de la locutrice, avec des montées de continuation majeures beaucoup plus amples qu'au début du récit. Dans ce récit particulier, la locutrice crée de surcroît un effet de liste sur 'ils avaient enlevé les lustres/et ils avaient aussi enlevé les ampoules/ils avaient aussi enlevé les douilles' qui apparaissent nettement sur les tracés prosodiques suivants :

Figure 3. Prosogram montrant l'effet de liste dans la phase de complication du récit.
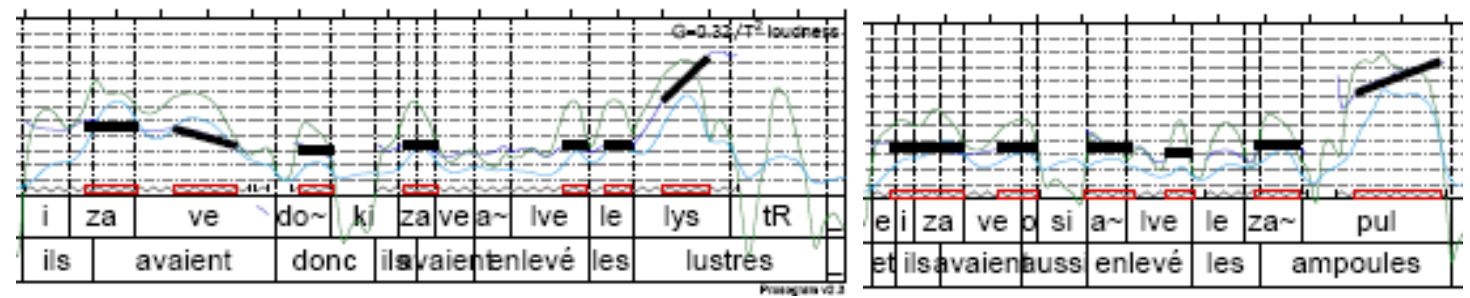

Cet effet de liste est par ailleurs renforcé par les deux gestes métaphoriques qui accompagnent le discours et avec lesquels la narratrice énumère les items verbaux sur l'index d'une main puis de l'autre, comme il apparaît dans la section suivante sur la gestualité.

C'est cependant l'apogée du récit qui attire notre attention puisque l'intensité et la F0 sont soudainement élevées vers la plage haute de la locutrice et restent élevées pendant toute cette phase du récit.

Figure 4. Prosogram montrant une intensité et une F0 élevées sur l'apogée du récit. 


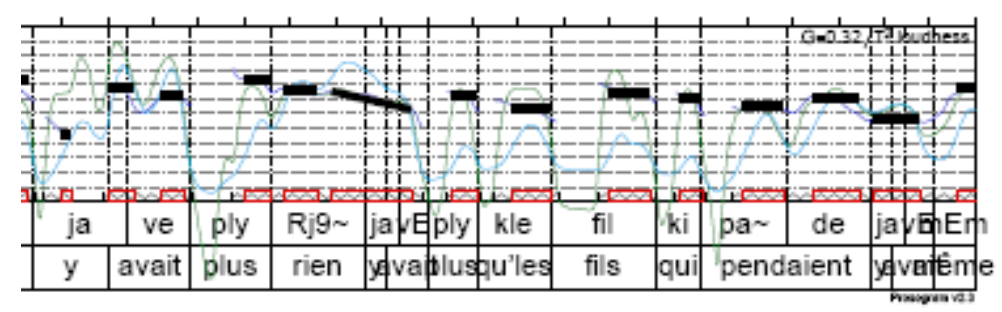

Dans les phases qui suivent l'apogée, les valeurs d'intensité et de F0 décroissent progressivement vers la plage basse de la locutrice et restent peu élevées jusqu'à la fin du récit :

Figure 5. Prosogram de la parenthèse du récit.

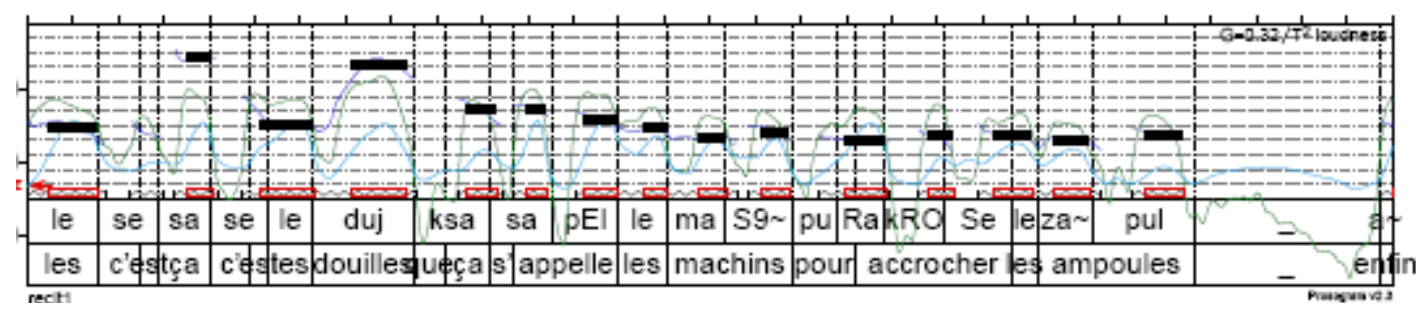

Ainsi qu'il a été mentionné au début de cette section, le débit de parole joue également un rôle, notamment dans la dramatisation du récit. Le débit de parole moyen pour cette locutrice sur tout le récit est de 6,5 syllabes par seconde (syll/sec), mais juste avant l'apogée, il augmente de manière drastique à $8,7 \mathrm{syll} / \mathrm{sec}$, après quoi il revient à la moyenne sur l'apogée du récit, puis augmente de nouveau à 8,6 syll/sec sur la parenthèse. On peut donc dire que l'apogée du récit se démarque des phases environnantes par une différence de tempo.

\subsection{Paramètres gestuels dans le récit}

En ce qui concerne les paramètres gestuels étudiés sur ce récit, on observe qu'à la fin de chaque phase structurelle, la narratrice regarde son interlocutrice. Ce regard orienté vers l'autre a une fonction phatique et l'interlocutrice qui regarde la narratrice tout au long du récit y répond par un hochement de tête, c'està-dire un backchannel gestuel (voir Bertrand et al., 2007). Au-delà des phases structurelles, la narratrice a également un regard phatique vers son interlocutrice à la fin de chaque événement de la phase de complication, où l'on trouve également des backchannels de l'interlocutrice. Des études précédentes ont montré que les backchannels sont produits de préférence après des points de complétude syntaxique, sémantique et prosodique (les Transition Relevance Places - TRPs - de Sacks et al., 1974, repris plus tard dans Couper-Kuhlen \& Selting, 1996, mais aussi Bertrand et al., 2007). Bien que la notion de TRP ait été utilisée différemment selon les auteurs, qui voient parfois une possible TRP dans la proposition syntaxique, il semble que dans les séquences narratives, le contenu sémantique joue un rôle majeur dans ce qui doit être entériné par l'interlocutrice. Le fait que les backchannels sont produits après chaque événement du récit et non pas après les propositions syntaxiques montre bien que l'événement constitue une unité sémantique complète du point de vue de la structure narrative et corrobore donc les unités et les phases décrites par Labov (op. cit.).

Les phases du récit apparaissent également dans les gestes produits par la narratrice. Comme il a été montré dans la section précédente sur la prosodie, la narratrice dans ce récit crée un effet de liste à la fin de la phase de complication ce qui contribue à augmenter la tension du récit jusqu'à l'apogée. Cet effet de liste est d'ailleurs renforcé par la gestualité de la narratrice : elle produit un mouvement de scansion de la tête (beat) sur 'ils avaient enlevé les lustres' et un deuxième sur 'ils avaient aussi enlevé les ampoules'. Les deux mouvements donnent de l'emphase aux deux événements, qui sont considérés comme deux items distincts dans le récit. Elle accompagne également chacun de ces deux événements de deux gestes 
métaphoriques grâce auxquels elle énumère les deux items sur ses doigts comme il apparaît dans les deux photos suivantes :

Figure 6. Deux métaphoriques exprimant l'énumération dans la phase de complication du récit.
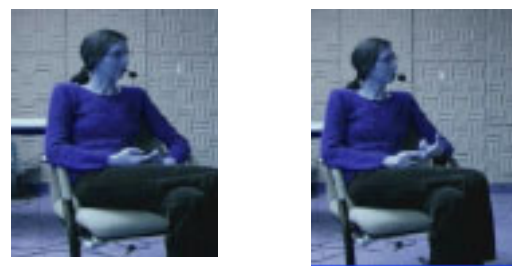

Sur l'apogée du récit, les auditeurs profanes ont remarqué le geste iconique produit par la locutrice qui effectue un mouvement vertical de la main gauche (pour illustrer les fils qui pendent du plafond). Ce geste particulier les a incités à penser que cette proposition devait constituer l'apogée du récit. On ne peut que recommander la prudence face à ce que rapportent les auditeurs ici, car l'annotation montre que la locutrice effectue d'autres gestes iconiques au cours de son récit: en fait, elle commence la phase d'orientation du récit avec un geste iconique (un geste horizontal des deux mains illustrant toutes les prises sur la même façade) et produit deux autres gestes iconiques (appuyer sur l'interrupteur, (dé)visser une ampoule, voir les photos a et $b$ de la Figure 7 plus bas) dans la phase de complication qui précède l'apogée. On ne peut également dire qu'une plus grande densité gestuelle sur l'apogée influencerait les auditeurs puisqu'un grand nombre de gestes sont produits sur les autres phases du récit. Je me suis donc demandée en quoi le geste iconique produit sur l'apogée retenait l'attention des auditeurs qui ne semblent pas remarquer les nombreux autres gestes produits au cours du récit. Si l'on compare ce geste aux autres iconiques, il apparaît que le mouvement réalisé par la locutrice est beaucoup plus ample. Elle lève la main au-dessus de sa tête et la descend au niveau son torse, alors que les autres gestes sont réalisés plus près du corps de la narratrice (ce que McNeill appelle 'the speaker's close periphery', 1992). De cette manière, la narratrice donne plus de visibilité à son geste, par rapport aux autres gestes du récit. Il est intéressant également de noter une progression dans l'ampleur des gestes réalisés (du geste le moins ample en début de narration au geste le plus ample sur l'apogée, voir Figure 7 ci-dessous), amplification en lien direct avec l'amplification prosodique décrite dans la section précédente.

Ce qui peut également contribuer à la bonne visibilité du geste est la main avec laquelle il est réalisé. McNeill (1992) a montré que la main utilisée par les locuteurs dans la réalisation des gestes avec une seule main dépend du locuteur et n'est pas nécessairement liée au fait que le locuteur soit droitier ou gaucher. Il a également montré que les locuteurs tendent pour ces gestes à utiliser la même main dominante mais pas de manière systématique. Il n'a pas exploré les contextes de changement de main pour ces gestes, ce qui s'avérerait être extrêmement complexe cependant. Tout en restant prudent sur l'interprétation car de nombreux facteurs peuvent intervenir dans l'utilisation de la main gauche ou de la main droite par les locuteurs, il se trouve que dans ce récit (ainsi que dans d'autres séquences narratives produites par des narratrices différentes), la narratrice réalise tous ses gestes simples avec la main droite dans le récit, excepté sur l'apogée où elle réalise un geste iconique et un geste métaphorique avec la main gauche. En terme de proxémique, l'interlocutrice est assise également à sa gauche, de sorte que l'utilisation de la main gauche par la locutrice rapproche son geste de son interlocutrice à qui il devient plus visible, alors que les gestes réalisés avec la main droite sont en fait réalisés plus loin de l'interlocutrice. On peut donc dire qu'en employant un geste réalisé plus près de l'interlocutrice et de manière plus ample, la narratrice montre une plus grande orientation vers l'interlocutrice, selon le terme employé par Harness Goodwin (1982). 
Figure 7. Gestes iconiques simples réalisés par la narratrice avec la main droite pendant la phase de complication ( $a$ et $b$ ) et avec la main gauche sur l'apogée du récit (c).

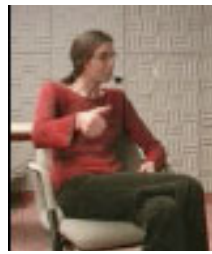

(a)

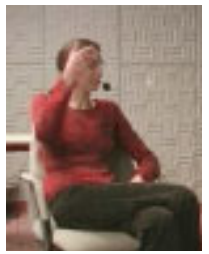

(b)

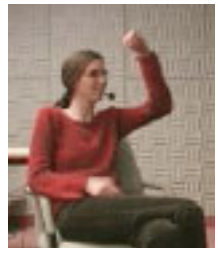

(c)

Ce qui ressort néanmoins est une certaine unité gestuelle dans le récit, que McNeill nomme « catchment » (McNeill, 2001; McNeill et al., 2001 a et b). Ce «catchment» consiste en une forme gestuelle récurrente :

\begin{abstract}
"A catchment is recognized when one or more of gesture features recur in at least two (not necessarily consecutive) gestures. The logic is that the recurrence of an image in the speaker's thinking will generate recurrent gesture features. Recurrent images suggest a common discourse theme. Then, working backwards, the recurring features offer clues to the cohesive linkages in the text with which it co-occurs. A catchment is a kind of thread of visuospatial imagery that runs through a discourse to reveal the larger discourse units that emerge out of otherwise separate parts." (McNeill et al., 2001a: 474).
\end{abstract}

Pour revenir au récit étudié ici, contrairement au récit précédent mentionné plus haut (concernant toutes les prises de téléphone installées sur le même mur) où l'unité gestuelle apparaissait à travers une série de gestes réalisés dans le plan horizontal de la locutrice, dans celui-ci, qui porte sur l'éclairage de l'appartement, tous les gestes iconiques sont réalisés dans un plan vertical. C'est ce plan unique qui confère l'unité gestuelle du récit, le « catchment ».

Enfin, la phase d'évaluation s'accompagne également de gestes spécifiques qui dépendent cependant du type de récit. Dans un récit humoristique comme dans l'exemple présenté ici (qui est aussi le type de récit le plus fréquent dans le corpus CID), l'évaluation est exprimée principalement par des expressions vocales et faciales comme les rires, les sourires et les négations de la tête. Cependant, alors que les deux mouvements de négation de la tête de cet exemple sont réalisés uniquement dans la phase d'évaluation pour porter un jugement négatif sur le comportement des propriétaires, les rires et les sourires apparaissent également dans des phases précédentes du récit (par exemple, la narratrice rit à la fin de la phase de complication juste avant l'apogée, rire qui trouve un écho dans le sourire subséquent de l'interlocutrice) et ils sont produits à la fois par la narratrice et par son interlocutrice. Cela signifie que les deux participantes anticipent la chute comique de l'anecdote. Elles réitèrent d'ailleurs les rires et les sourires dans la phase d'évaluation. Il faut voir également que les rires/sourires et les mouvements de tête jouent deux rôles différents dans l'interaction: dans leur étude sur les backchannels, Bertrand et al. (op. cit.) considèrent que les rires/sourires et les mouvements de tête peuvent être produits par l'interlocuteur comme backchannels avec un sens de assessment, c'est-à-dire une évaluation de ce qui vient d'être dit. Lorsqu'ils accompagnent une évaluation verbale (par exemple dans la phase d'évaluation d'un récit), leur fonction ne se limite plus uniquement à la paire phatique/backchannel qu'ils peuvent avoir dans d'autres phases du récit, car ils portent alors un plus grand poids évaluatif. Ainsi, alors que Labov considère l'évaluation comme une production verbale du narrateur, on peut étendre la notion d'évaluation aux productions et comportements de l'ensemble des participants à l'interaction, comme révélatrice de leur orientation vis-à-vis du récit (ainsi, que le proposent C. et M.H. Goodwin dans leurs travaux cités plus haut). 


\section{Conclusion}

Ce qui est apparu dans cet article à travers l'étude de cas d'un récit est que la séquence narrative constitue une action en elle-même qui implique un faisceau de paramètres, pris en compte par les participants à l'interaction, même de manière inconsciente. Dans l'exemple présenté ici, certains paramètres prosodiques se sont révélés essentiels dans la structuration du récit, et à la création d'une tension avant l'apogée, c'est-à-dire l'événement le plus important du récit, par des montées de continuation majeures et une accélération du tempo. L'apogée elle-même est réalisée avec une augmentation de l'intensité et de la F0 ainsi qu'un ralentissement du débit de parole. Ces observations convergent avec les résultats observés par Auer dans Couper-Kuhlen \& Selting (1996), même si son étude portait sur des unités discursives plus petites. La gestualité joue également un rôle dans les interactions et est dans la narration en accord avec les paramètres prosodiques puisqu'il est apparu dans l'exemple cité que les gestes produits sur l'apogée sont plus visibles pour l'interlocutrice puisqu'ils sont réalisés plus amplement que les autres gestes du récit mais également plus près de l'interlocutrice.

Un autre point essentiel de cette étude est que les paramètres acoustiques et visuels corroborent le travail pionnier de Labov \& Waletzky sur la structure verbale des récits, ainsi que toutes les autres études qui s'inscrivent dans ce cadre de l'analyse du discours, bien que, me semble-t-il, aucune étude sur le récit n'ait encore pris en compte les paramètres prosodiques et gestuels de manière systématique, alors qu'ils sont pourtant à la base de toute interaction en face-à-face (voir les travaux cités en introduction). En effet, par opposition au récit textuel, l'oralité des récits conversationnels est marquée par leur structure verbale, mais aussi leur forme prosodique (intonation, intensité et débit de parole moyens dans la phase d'orientation, contours intonatifs montants pour marquer la succession des événements, accélération du débit de parole juste avant l'apogée du récit, F0 et intensité plus élevées sur l'apogée, et déclin des paramètres acoustiques sur la phase d'évaluation). Dans ce corpus, les récits ont également une dimension visuelle en harmonie avec la dimension orale : au cours du récit, les gestes se font plus amples pour atteindre leur extension maximale sur l'apogée. Au moment de produire cette apogée, le narrateur montre une plus grande orientation vers l'auditeur (par le regard, par le rire qui incite l'autre à rire également, mais aussi en produisant un geste manuel plus proche de l'auditeur que les autres gestes réalisés dans le récit). L'unité du récit, quant à elle, est marquée par la caractéristique commune des gestes iconiques (ici, le fait qu'ils soient réalisés dans un plan vertical).

Cependant, il faut aussi noter que tous les récits ne présentent pas la même structure verbale canonique. Par exemple, il est apparu qu'aucun des récits étudiés sur le corpus ne comprenait l'abstract décrit par Labov \& Waletzky et que la coda est très rare, ce qui ne remet pas nécessairement en cause les travaux antérieurs. Il existe de nombreux types d'interaction et de méthodes d'élicitation des séquences narratives, ce qui donne naturellement différent types de structures narratives. Ce qui est vrai dans un contexte particulier d'interaction peut ne pas être observé dans une situation d'enregistrement légèrement différente. De plus, les paramètres prosodiques et gestuels montrent une grande variabilité intra- et interlocuteur dans l'usage qu'en font les participants à l'interaction, de même que dans la manière dont ils sont perçus par les auditeurs. Cela ne signifie pas qu'ils ne jouent pas un rôle important dans l'interaction, mais simplement que les chercheurs doivent être peut-être plus prudents dans leur interprétation. Le but de l'analyse du rôle de ces paramètres dans une étude de cas sur les séquences narratives est de donner un aperçu de la manière dont les paramètres sont utilisés par les interactants mais aussi d'orienter de futures recherches sur les paramètres qui se sont avérés jouer un rôle dans la structuration du récit et qui fonctionneront peut-être de manière similaire dans des récits différents. En revanche, il ne serait pas raisonnable à ce stade de la recherche scientifique internationale sur le récit de dire que tous les locuteurs utilisent les paramètres prosodiques et gestuels de la même manière lorsqu'ils racontent un récit.

Pour finir sur une remarque plus optimiste, bien qu'un seul récit ait été présenté dans cet article pour donner une idée plus précise de la façon dont les dimensions verbales, vocales et gestuelles sont utilisées conjointement par les locuteurs dans les interactions, la recherche de l'auteur sur le récit est basée sur une plus grande quantité de séquences, produites au total par six jeunes femmes, anglaises et françaises. La plupart des observations faites sur l'exemple donné ici pour les différents paramètres prosodiques et gestuels ont également pu être faites sur d'autres récits produits par d'autres locutrices. On peut donc 
espérer la publication d'un nouvel article prochainement, qui reprendra les indices audio-visuels systématiquement utilisés par les narratrices occidentales.

\section{Références bibliographiques}

Bamberg, M. (Ed.). (2007). Narrative - State of the Art. Amsterdam, Philadelphia : John Benjamins.

Bertrand, R., P. Blache, R. Espesser, G. Ferré, C. Meunier, B. Priego-Valverde, and S. Rauzy. (2006). Le CID Corpus of Interactional Data - : protocoles, conventions, annotations. Travaux Interdisciplinaires du Laboratoire Parole et Langage (TIPA) 25, 31-60.

Bertrand, R., Ferré, G., Espesser, R., Rauzy, S., and Blache, P. (2007). Backchannels revisited from a multimodal perspective. Proceedings of Auditory-visual Speech Processing (2007 August 31-September 3 : Hilvarenbeek, The Netherlands). Non paginé.

Bouvet, D. and Morel M.-A. (2002). Le ballet et la musique de la parole. Le geste et l'intonation dans le dialogue oral en français. Bibliothèque des Faits de Langues, Paris, Gap: Ophrys.

Chafe, W. (1997). Polyphonic Topic Development. In T. Givón (Ed.), Conversation. Cognitive, Communicative and Social Perspective 34. Amsterdam, Philadelphia : John Benjamins, 41-53.

Coates, J. (1997). The Construction of a Collaborative Floor in Women's Friendly Talk. In T. Givón (Ed.), Conversation. Cognitive, Communicative and Social Perspective 34. Amsterdam, Philadelphia : John Benjamins, 55-89.

Coates, J. (2001). "My mind is with you": Story sequences in the talk of male friends. Narrative Inquiry 11 (1), 81101.

Couper-Kuhlen, E. and M. Selting. (1996). Prosody in Conversation. Cambridge : CUP.

Ervin-Tripp, S. M. and A. Küntay. (1997). The Occasioning and Structure of Conversational Stories. In T. Givón (Ed.), Conversation. Cognitive, Communicative and Social Perspective 34. Amsterdam, Philadelphia : John Benjamins, 133-166.

Ferré, G. (2005). Gesture, Intonation and the Pragmatic Structure of Narratives in British English Conversation. York Papers in Linguistics 2, 55-90.

Ferré G., Bertrand, R., Blache, P., Espesser R., and Rauzy S. (2007). Intensive Gestures in French and their Multimodal Correlates, Proceedings of Interspeech, Antwerp, Belgium, 690-693.

Goodwin, C. (1984). Notes on Story Structure and the Organization of Participation. In J. Maxwell Atkinson and J. Heritage (Eds.), Structures of Social Action. Studies in Conversation Analysis. 225-246. New York: Cambridge University Press.

Goodwin, C. (1986). Audience Diversity, Participation and Interpretation. Text 6 (3), 283-316.

Goodwin, C. and Harness Goodwin M. (1992). Context, Activity and Participation. In P. Auer \& A. Di Luzio (Eds.), The Contextualization of Language. Amsterdam, Philadelphia : John Benjamins, 76-99.

Harness Goodwin, M. (1982). "Instigating": storytelling as social process. American Ethnologist 60 (1), 799-819.

Harness Goodwin, M. (1997). Byplay: Negotiating Evaluation in Storytelling. In C. Feagin, G.R. Guy, D. Schiffrin \& J. Baugh (Eds.), Towards a Social Science of Language: Papers in Honor of William Labov 2: Social Interaction and Discourse Structures. Amsterdam, Philadelphia : John Benjamins, 77-102.

Küntay, A. and S. Ervin-Tripp. (1997). Conversational Narratives of Children: Occasions and Structures. Journal of Narrative and Life History 7,113-120.

Labov, W. (1997). Some Further Steps in Narrative Analysis. Journal of Narrative and Life History 7,: 395-415.

Labov, W. (2007). Narrative pre-construction. In M. Bamberg (Ed.), Narrative - State of the Art. Amsterdam, Philadelphia : John Benjamins, 47-56.

Labov, W. and J. Waletzky. (1967). Narrative analysis: Oral versions of personal experience. In J. Helm (Ed.), Essays on the verbal and visual arts: Proceedings of the 1966 Annual Spring Meeting of the American Ethnological Society. Seattle : University of Washington Press, 12-44. 
Lerner, G. H. (1992). Assisted Storytelling: Deploying Shared Knowledge as a Practical Matter. Qualitative Sociology 15 (3), 247-271.

Local, J. (2007). Phonetic Detail and the Organisation of Talk-in-Interaction. In Proceedings of ICPhS XVI, Saarbrücken, 6-10 August 2007. Paper ID 1785.

McNeill, D. (1992). Hand and Mind: What Gestures Reveal about Thought. Chicago, London : The University of Chicago Press.

McNeill, D. (2001). Growth points and catchments. In C. Cavé, I. Guaïtella, and S. Santi (Eds.), Oralité et Gestualité (ORAGE) : "Interactions et comportements multimodaux dans la communication", Paris, L'Harmattan, 25-33.

McNeill, D., Quek F., McCullough K.E., Duncan S., Furuyama N., Bryll R., and Ansari R. (2001a). Catchments, prosody and discourse. In C. Cavé, I. Guaïtella, and S. Santi (Eds.), Oralité et Gestualité (ORAGE) : "Interactions et comportements multimodaux dans la communication", Paris, L'Harmattan, 474-481.

McNeill, D., Quek F., McCullough K.E., Duncan S., Furuyama N., Bryll R., Ma X.F., and Ansari R. (2001b). Catchments, prosody and discourse. Gesture 1(1), 9-33.

Ochs, E. (1997). Narrative. In Teun Van Dijk (Ed.), Discourse as Structure and Process. London : Sage Publications, 185-207.

Ochs, E. and L. Capps. (1997). Narrative Authenticity. Journal of Narrative and Life History 7, 83-89.

Portes C.and Bertrand R. (2006). Some cues about the interactional value of the 'continuation' contour in French, Actes Discours et Prosodie comme Interface Complexe (IDP), Cederom.

Portes, C., R. Bertrand, and R. Espesser. (2007). Contribution to a grammar of intonation in French. Nouveaux Cahiers de Linguistique Française 28, 155-162.

Sacks, H. (1995). Lectures on conversation : volumes I \& II, ed. by G. Jefferson; with an introduction by E. A. Schegloff. Oxford : Blackwells.

Sacks, H., E. Schegloff, and G. Jefferson. (1974). A simplest systematics for the organisation of turn-taking in conversation. Language 50, 696-735.

Sacks, H. and Schegloff, E.. (2002). Home Position. Gesture 2 (2), 133-146.

Selting, M. (1992). Intonation as a Contextualization device: Case Studies on the Role of Prosody, Especially Intonation, in Contextualizing Story Telling in Conversation. In Peter Auer and Aldo di Luzio (Ed.), The Contextualization of Language. Amsterdam, Philadelphia : John Benjamins, 233-258.

Taylor, S. (2007). Narrative as construction and discursive resource. In M. Bamberg (Ed.), Narrative - State of the Art. Amsterdam, Philadelphia : John Benjamins, 113-122.

Trabasso, T. and A. Özyürek. (1997). Communicating Evaluation in Narrative Understanding. In T. Givón (Ed.), Conversation. Cognitive, Communicative and Social Perspective 34. Amsterdam, Philadelphia : John Benjamins, 269-302.

\section{Outils}

Praat (Boersma P. and D. Weenick): A system for doing phonetics by computer. http://www.praat.org

EasyAlign (J.-P. Goldman): http://latlcui.unige.ch/phonetique/

Elan (H. Sloetjes): http://www.lat-mpi.eu/tools/elan/

Prosogram (P. Mertens): http://bach.arts.kuleuven.be/pmertens/prosogram/

\section{Remerciements}

Je remercie tout particulièrement $\mathrm{P}$. Blache et $\mathrm{R}$. Bertrand pour m'avoir autorisée à travailler sur ce corpus. Je tiens également à remercier les participants qui ont accepté de voir leur photographie publiée dans un article de recherche. Je remercie enfin les deux relecteurs anonymes qui, par leurs commentaires judicieux, ont largement contribué à améliorer cet article. 
${ }^{1}$ Certains auteurs dans Bamberg (2007) s'interrogent d'ailleurs sur la véracité des récits tirés de l'expérience personelle du narrateur.

${ }^{2}$ Si le sonore et le visuel sont tout à fait intégrés dans le discours, il est difficile, pour des raisons de clarté dans l'analyse, de ne pas «séparer» artificiellement ce qui est de l'ordre du sonore de ce qui touche le visuel. Cette séparation arbitraire permet de faire ressortir, entre autres choses, l'unité prosodique ou gestuelle d'une séquence, que celle-ci soit narrative (comme c'est le cas ici) ou explicative, monologique ou dialogique.

${ }^{3}$ Par «multimodale », j'entends une approche qui prend en compte la modalité orale (par l'analyse du verbal et du vocal, c'est-à-dire du discours et de la prosodie) et la modalité visuelle (par l'analyse des gestes co-verbaux et des mouvements des participants).

${ }^{4}$ Par ce terme, j'entends des récits produits lors de la conversation. L'analyse qui en est faite s'inscrit dans un cadre d'analyse du discours et non pas dans le cadre de l'Analyse Conversationnelle. 